\title{
Morphometric Analysis for Pathological Abnormality Detection in the Skull Vaults of Adolescent Idiopathic Scoliosis Girls
}

\author{
Lin Shi ${ }^{1,2}$, Pheng Ann Heng ${ }^{1,2}$, Tien-Tsin Wong ${ }^{1,2}$, Winnie C.W. Chu ${ }^{3}$, \\ Benson H.Y. Yeung ${ }^{4}$, and Jack C.Y. Cheng ${ }^{4}$
}

${ }^{1}$ Department of Computer Science and Engineering, The Chinese University of Hong Kong, Shatin, N.T., Hong Kong, China

\{1shi, pheng, ttwong\}@cse.cuhk.edu.hk

${ }^{2}$ Shun Hing Institute of Advanced Engineering, The Chinese University of Hong

Kong, Shatin, N.T., Hong Kong, China

${ }^{3}$ Department of Diagnostic Radiology and Organ Imaging, The Chinese University of Hong Kong, Shatin, N.T., Hong Kong, China

winnie@ruby.med.cuhk.edu.hk

${ }^{4}$ Department of Orthopaedics and Traumatology, The Chinese University of Hong

Kong, Shatin, N.T., Hong Kong, China

\{byeung, jackcheng\}@cuhk.edu.hk

\begin{abstract}
In this paper, we present a comprehensive framework to detect morphological changes in skull vaults of adolescent idiopathic scoliosis girls. To our knowledge, this is the first attempt to use a combination of medical knowledge, image analysis techniques, statistical learning tools, and scientific visualization methods to detect skull morphological changes. The shape analysis starts from a reliable 3-D segmentation of the skull using thresholding and math-morphological operations. The gradient vector flow is used to model the skull vault surface, which is followed by a spherically uniform sampling. The scale-normalized distances from the shape centroid to sample points are defined as the features. The most discriminative features are selected using recursive feature elimination for support vector machine. The results of this study specify the skull vault surface changes and shed light on building the evidence of bone formation abnormality in AIS girls.
\end{abstract}

\section{Introduction}

Adolescent idiopathic scoliosis (AIS) is a cause-unknown complicated 3-D spine deformity appearing most commonly in girls between 10 and 16 years old. The current treatment of AIS is still unsatisfactory because the etiopathogenesis remains unclear 1. To enable evidence-based treatments and prognosis of curve progression, research on AIS has been carried out from various aspects, e.g. neuromotor mechanisms, hormonal system, and genetics [2]. In previous research studies carried out by our center, we have found persistently low bone mineral density 345678 and abnormal skeletal growth in AIS during peri-pubertal 
development, which includes both peripheral [9] and axial skeleton [1011]. Compared with age-matched controls, the longitudinal growth of the vertebral bodies in AIS is disproportionate and faster and mainly occurs by endochondral ossification. In contrast, the circumferential growth by membranous ossification is slower in both the vertebral bodies and pedicles 10. We have hypothesized that there is generalized abnormal systemic growth in AIS and there might be associated abnormal growth and ossification of whole skull, which comprises the endochondral and membranous ossification process. In this work, we sought to analyze skull vault morphologies, which might bring out inspiring findings of ossification abnormality of the skull in AIS girls.

In a typical shape analysis application, the first step is the acquisition of the 2-D or 3-D shape from the data; it usually refers to image segmentation. Then shape descriptors are extracted to quantitatively characterize the shape. Finally, the shape difference accounts for the classification can be inferred from statistical analysis performed on these numerical descriptors.

Shape Extraction. There is limited work on segmenting skulls from MRI data, since the signals of bony structures is very weak in MRI. In recent work of Dogdas et al., 12, a human skull and scalp segmentation method is proposed based on thresholding and morphological operations.

Shape Description. Driven by various applications, shape description methods include parametric methods, e.g., spherical harmonic transform [13, and nonparametric methods, e.g., distance transform [14. Snakes have been used to represent an object boundary as a parameter curve or surface. Xu et al. proposed the gradient vector flow (GVF) snake [15], which addresses the problems of the short capture range and inability to track at boundary concavity.

Statistical Analysis. The determination of discriminative descriptors that separates the two groups with least error can be interpreted as a feature selection problem in the machine learning domain. Feature selection methods can be classified into three categories [16], i.e., filter, wrapper, and embedded approaches. Various feature selection schemes have been proposed for the popular large margin classifier, support vector machine (SVM). Guyon et al. [16] introduced SVMRFE, a backward elimination method for gene selection using SVM.

Figure 1 shows the framework of the proposed skull vault analysis scheme. The implementation details are introduced in the remainder of this paper.

\section{Skull Vault Extraction and Description}

\section{$2.1 \quad$ Skull Segmentation}

Reliable 3-D skull segmentation is an indispensable prerequisite for further feature extraction and statistical analysis. However, defining the skull in MRI is a challenging problem because of the weak magnetic resonance signals in the bony structures. We adopt a recently proposed effective strategy for skull segmentation in MRI data [12, which uses simple thresholding and math-morphological operations but achieves results close to CT data, especially in the range of skull vault. Before segmentation, the images are first preprocessed using the anisotropic 

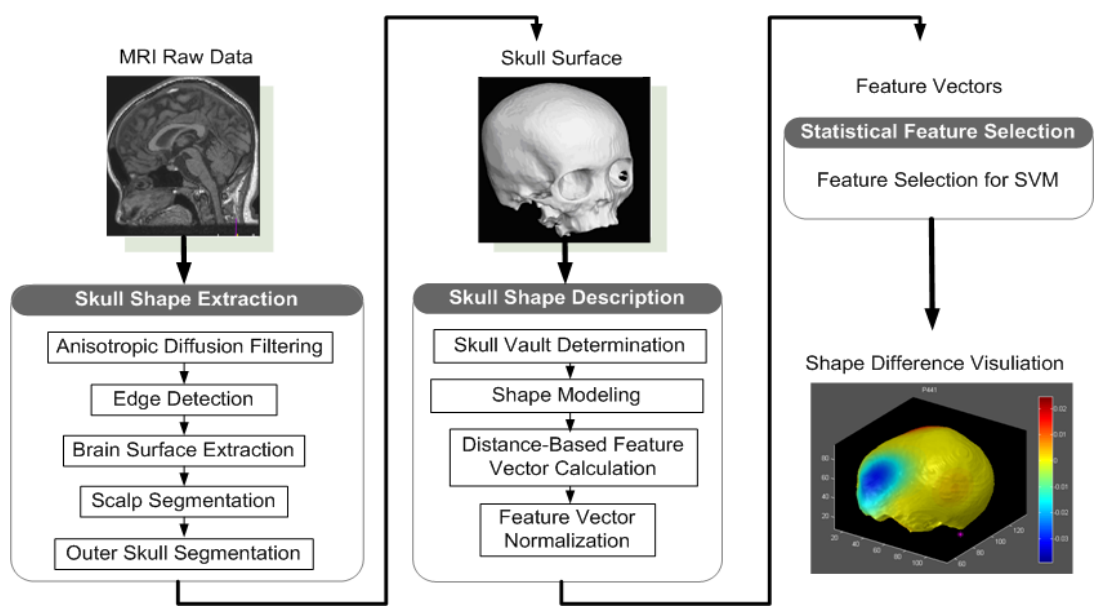

Fig. 1. The framework of skull vaults difference learning proposed in this paper

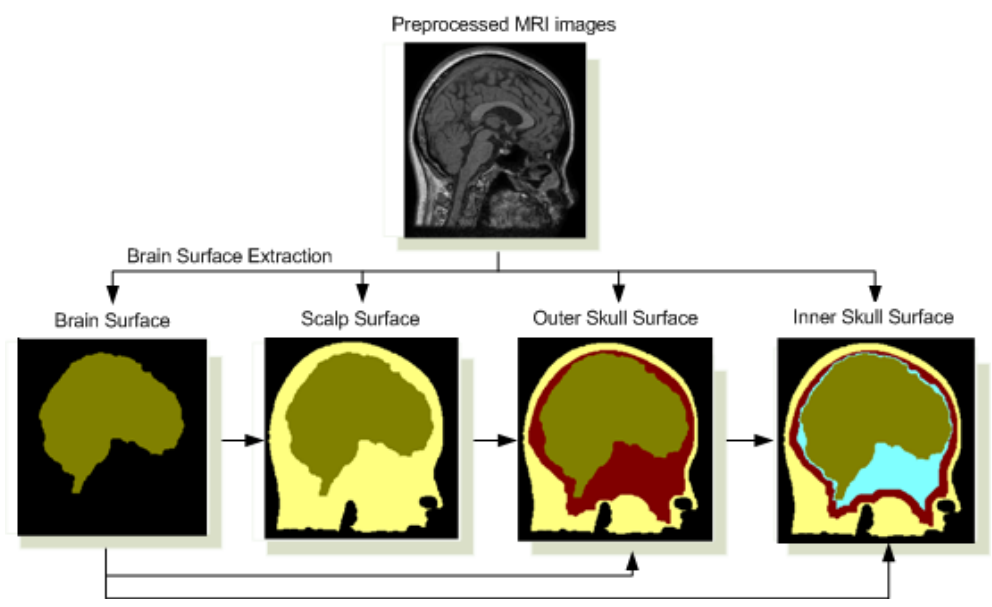

Fig. 2. The process of skull segmentation using thresholding and 3-D mathmorphological operations

diffusion filter, which smoothes the volume while preserving sharp edges. The brain is extracted using the BSE algorithm [17. The segmented brain is used as an inner bound for further skull segmentation. Thereafter, the scalp, the outer bound of the skull, is segmented using a combination of thresholding and mathmorphological operations. Then the skull surface is found between outlines of brain surface and scalp using thresholding and morphological closing and opening. Figure 2 gives a summary of the skull segmentation process.

\subsection{Skull Vault Description}

In our particular application, we are interested in localizing the discriminative features in the skull vaults between AIS patients and normal controls, thus local 
descriptors are preferred, which implies straightforward correspondences with the spatial distribution of the skull vault.

Skull Vault Determination. The skull vault is normally measured in anthropometry as the upper part of the skull determined by the plane passing through the three well-defined anatomical landmarks: bilateral frontozygomatic synchondrosis and the most superior posterior point of the external auditory meatus, as shown in Figure 4 (a). In order to be accurate, we identify the three landmarks on the 2-D slices. In Figure 3 (a), we find the frontozygomatic synchondrosis on the axial slice where the neighboring slices have significant morphological changes pointed by the thick arrows. The most superior posterior point of the external auditory meatus is determined as shown in Figure 3(b). The separated skull vault and the separating plane are reconstructed in Figure 4 (b).

Skull Vault Modeling. Given the edge map $f(\mathbf{x}): \Omega \rightarrow \mathbb{R}$ defined in $\Omega \subset \mathbb{R}^{3}$, the three-dimensional GVF field is defined as the vector field $\mathbf{v}(s): \Omega \rightarrow \mathbb{R}^{3}$ that minimizes the energy function

$$
\epsilon=\int_{\Omega}\left(\mu|\nabla \mathbf{v}|^{2}+|\nabla f|^{2}|\mathbf{v}-\nabla f|^{2}\right) d \mathbf{s},
$$

where $\mu$ is the regularization parameter and $f$ is the edge map determined using the edges on the image convoluted with a Gaussian kernel. The GVF field can be obtained by solving the Euler equations

$$
\mu \nabla^{2} \mathbf{v}-(\mathbf{v}-\nabla f)|\nabla f|^{2}=0,
$$

where $\nabla^{2}$ is also applied to each component of the vector field $\mathbf{v}$ separately.

Distance-Based Feature Calculation. Using GVF, a smooth description of the surface is obtained. A uniformly sampled sphere (see Figure 5 (a)) is generated for each reconstructed skull vault model $m_{i}(i=1, \cdots,|S|)$, where $S$ is

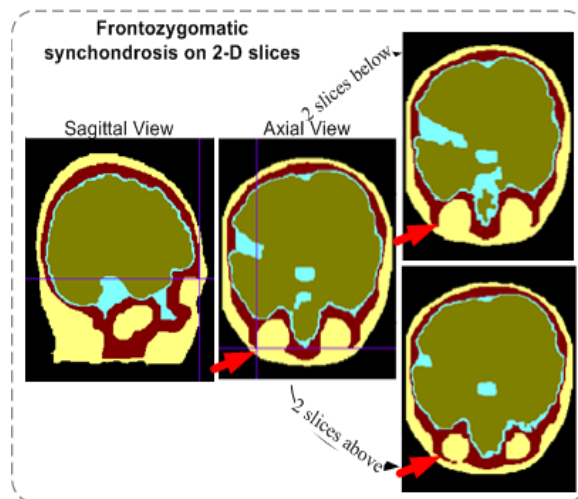

(a)

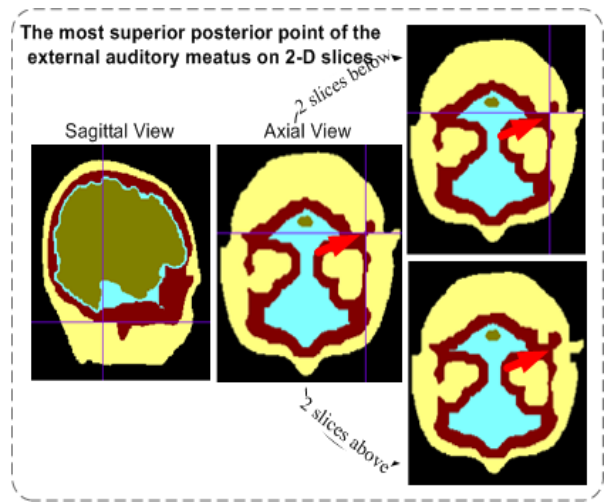

(b)

Fig. 3. Identification of the landmarks frontozygomatic synchondrosis, the most superior posterior point of the external auditory meatus that determine the skull vault demarcation plane. (a) Finding frontozygomatic synchondrosis. (b) Finding the most superior posterior point of the external auditory meatus. 


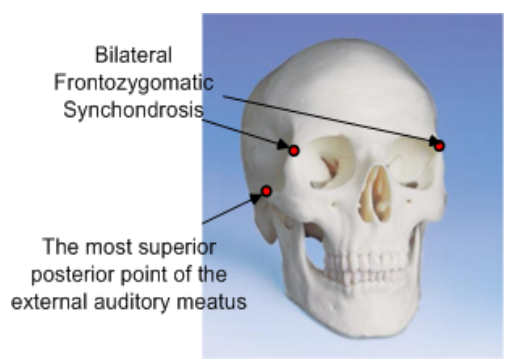

(a)

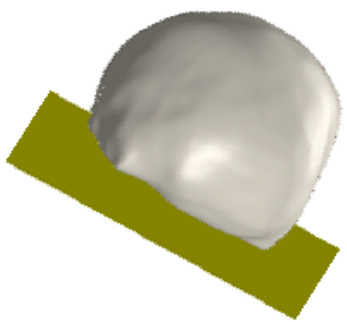

(b)

Fig. 4. Separation of the skull vault from the whole skull. (a) Positions of the three landmarks on a real skull model indicated by the red points. (b) The skull vault determined by the separating plane passing through the three anatomical landmarks.

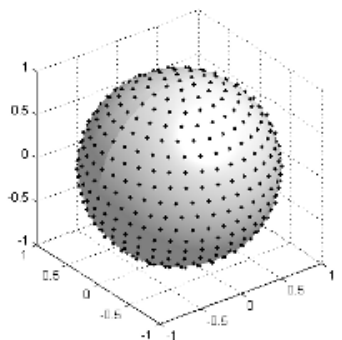

(a)

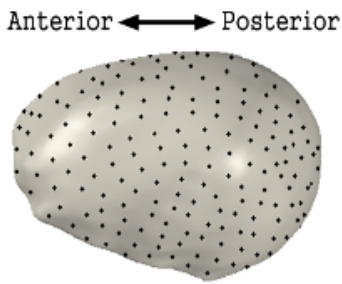

(b)

Fig. 5. Distance-based feature calculation. (a) The unit sphere centered at the skull vault centroid. (b) The spherically uniform sample points on the skull vault surface.

the set containing all subjects, and $N$ points are sampled on each sphere. The center of sphere is translated to the centroid of $m_{i}$, and the positive direction along the $\mathrm{y}$ axis is rotated to the normal direction of the basal plane (or the separating plane defined in Figure 4) of $m_{i}$. Along each of these $N$ directions, a "ray" is emitted from the center and possibly intersects with $m_{i}$. These intersections are recorded as the candidate sample points for model $m_{i}$. For each skull vault model $m_{i}$, we can find that the sample points are obtained in the same way and inherently correspond to each other. In order to ensure equal length of the resultant feature vectors, the directions that have no intersection with the surface are tagged and the corresponding sample points are removed for all subjects in $S$. Then the distances from the remaining sample points (see Figure 5 (b)) to the centroid of $m_{i}$ are calculated as the features to describe the skull vault surface for subject $m_{i}$. These features are translationally invariant since they are all calculated relative to the centroid. And the rotational invariance is guaranteed because the basal planes have been implicitly aligned. In order for these features to be invariant to scaling, we normalize the feature vector for each subject $m_{i}$ as $\mathbf{x}_{i}^{\prime}=\frac{\mathbf{x}_{i}}{\left\|\mathbf{x}_{i}\right\|}$. 


\section{Pathological Abnormality Discovery Using Statistical Feature Selection}

In order to select the discriminative features, we adopt a popular wrapper feature selection method, the recursive feature elimination (RFE) for support vector machine (SVM) proposed by Guyon et al. [16 to solve a cancer gene classification problem. SVM-RFE performs feature selection by iteratively training an SVM with the current set of features and removing the feature with the smallest weight in the resulting hyperplane at a time. Ultimately, this algorithm results in a ranking list of features. The number of dominating features is selected by plotting a curve of the number of features against the accuracy, and choose the number of features to be relatively small but corresponds to the highest accuracy.

\section{Subjects and Results}

Subjects. In the current study, the MRI data set are acquired from 10 AIS patients between 12 and 18 years old and 10 age-matched healthy girls recruited from the Scoliosis Clinic of Prince of Wales Hospital, Hong Kong. The MRI scanner used is a $1.5 \mathrm{~T}$ MR scanner (Sonata, Siemens, Munich, Germany). The whole skull is scanned from left to right using 2-D acquisition and turbo spinecho $\mathrm{T} 2$-weighted sequence with $\mathrm{TR}=6500, \mathrm{TE}=118 \mathrm{~ms}$. The thickness is $3 \mathrm{~mm}$ without gap, and the field of view is $512 \times 512$.

Results. The effectiveness of the feature selection method is validated by the quality of the selected feature subsets in the classification. Figure 6 (a) shows the classification accuracies calculated as 1 minus the leave-one-out (LOO) errors given different numbers of selected features. One can find that the SVM-RFE selects a small feature subset which can classify AIS patients from normal controls with the highest accuracy. The classification accuracy begins to drop when too many features are added to the subset.

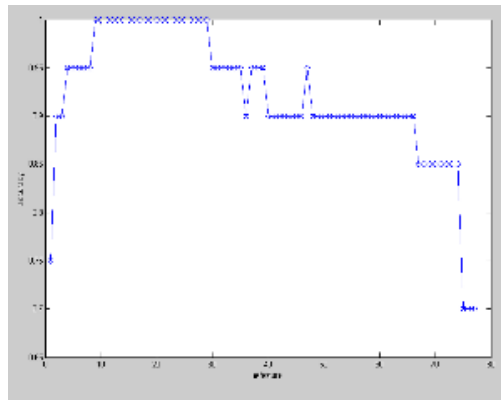

(a)

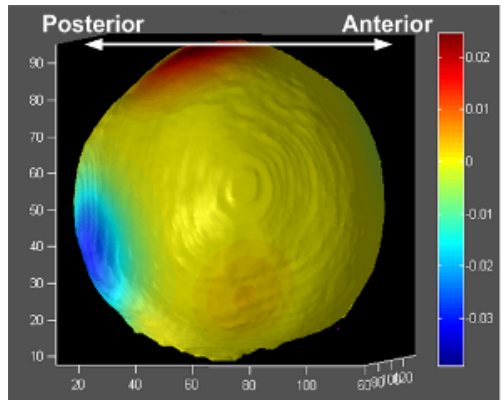

(b)

Fig. 6. (a) The classification accuracies given different sizes of selected dominating features. (b) The group difference in skull vaults between normal controls and AIS patients mapped on the skull vault of a randomly selected normal control: top view. 
The detected group difference in the skull vaults between normal controls and AIS patients are mapped on the skull vault of a randomly selected normal subject, as displayed in Figure 6 (b). The colors correspond to the values of difference: equal features for AIS and normal control are indicated by yellow; red means normal controls are statistically larger with respect to that particular feature; and blue means reversely. From the result, a direct observation is the posterior region in AIS is comparatively smaller while the left part is larger. Although it deserves further anatomical interpretations, this finding sheds light on building quantitative evidence of the ossification abnormality in AIS patients.

\section{Conclusion and Future Work}

This paper presents a comprehensive framework of the statistical morphology analysis for the detection of shape difference in the skull vaults between AIS patients and normal controls. To solve this particular problem, medical knowledge is combined with a variety of engineering techniques in areas of image processing, machine learning, and computer graphics. The preliminary detection result on a relatively small sample size (10 subjects in each group) is inspiring and encouraging. Based on these findings, it is promising to derive concrete evidence of systematic imbalance in the bone formation mechanisms in the AIS group.

As a pilot study, there is a lot to be explored in the future. Firstly, we plan to increase the number of subjects for more statistically reliable results. We realize that to form a clear interpretation of the current result, related findings and existing theories in other domains need to be considered. Meanwhile, the Cobb's angle of each subject, which quantifies the severity of scoliosis, can be used as the weight in statistical learning. The above work is part of an ongoing research project in the AIS Research Team using MR imaging of the whole central nervous system, which includes the skull, brain, vertebral column and spinal cord. We sought to investigate any disproportional growth between the skeletal (skull and vertebral column) and neural system (brain and spinal cord) and define the presence of neuro-osseous link in the etiopathogenesis of AIS.

\section{Acknowledgement}

The work described in this paper was supported by grants from the Research Grants Council of the Hong Kong Special Administrative Region (Project no. CUHK4223/04E and CUHK4506/05M) and CUHK Shun Hing Institute of Advanced Engineering.

\section{References}

1. Miller, N.H.: Cause and natural history of adolescent idiopathic scoliosis. Orthop Clin North Am 30 (1999) 343-52

2. Yeung, H.: Abnormal Skeletal Growth and Bone Remodeling in Adolescent Idiopathic Scoliosis - A Morphological and Genetic Study. PhD thesis, The Chinese University of Hong Kong (2006) 
3. Cheng, J., Qin, L., Cheung, C., Sher, A., Lee, K., Ng, S., Guo, X.: Generalized low areal and volumetric bone mineral density in adolescent idiopathic scoliosis. J Bone Miner Res. 15 (Aug. 2000) 1587-95

4. Cheng, J., Tang, S., Guo, X., Chan, C., Qin, L.: Osteopenia in adolescent idiopathic scoliosis: a histomorphometric study. Spine 26 (Feb. 2001) E19-23

5. Lee, W., Cheung, C., Tse, Y., Guo, X., Qin, L., Ho, S., Lau, J., Cheng, J.: Generalized low bone mass of girls with adolescent idiopathic scoliosis is related to inadequate calcium intake and weight bearing physical activity in peripubertal period. Osteoporos Int. 16 (Sep. 2005) 1024-35

6. Lee, W., Cheung, C., Tse, Y., Guo, X., Qin, L., Lam, T., Ng, B., Cheng, J.: Association of osteopenia with curve severity in adolescent idiopathic scoliosis: a study of 919 girls. Osteoporos Int. 16 (Dec. 2005) 1924-32

7. Hung, V., Qin, L., Cheung, C., Lam, T., Ng, B., Tse, Y., Guo, X., Lee, K., Cheng, J.: Osteopenia: a new prognostic factor of curve progression in adolescent idiopathic scoliosis. J Bone Joint Surg Am. 87 (Dec. 2005) 2709-16

8. Cheung, C., Lee, W., Tse, Y., Lee, K., Guo, X., Qin, L., Cheng, J.: Generalized osteopenia in adolescent idiopathic scoliosis-association with abnormal pubertal growth, bone turnover, and calcium intake? Spine 31 (Feb. 2006) 330-8

9. Cheung, C., Lee, W., Tse, Y., Tang, S., Lee, K., Guo, X., Qin, L., Cheng, J.: Abnormal peri-pubertal anthropometric measurements and growth pattern in adolescent idiopathic scoliosis: a study of 598 patients. Spine 28 (2003) 2152-2157

10. Guo, X., Chau, W., Chan, Y., Cheng, J.: Relative anterior spinal overgrowth in adolescent idiopathic scoliosis. results of disproportionate endochondral-membranous bone growth. J Bone Joint Surg Br. 85 (Sep. 2003) 1026-31

11. Guo, X., Chau, W., Chan, Y., Cheng, J., Burwell, R., Dangerfield, P.: Relative anterior spinal overgrowth in adolescent idiopathic scoliosisresult of disproportionate endochondral-membranous bone growth? summary of an electronic focus group debate of the ibse. Eur Spine J. 14 (Nov. 2005) 862-73

12. Dogdas, B., Shattuck, D.W., Leahy, R.M.: Segmentation of skull and scalp in 3-d human mri using mathematical morphology. Human Brain Mapping 26 (2005) 273-85

13. Brechbühler, C., Gerig, G., Kübler, O.: Parameterization of closed surfaces for 3-d shape description. CVGIP: Image Understanding 61 (1995) 154-70

14. Golland, P., Grimson, W., Shenton, M.E., Kikinis, R.: Small sampling size for shape analysis of anatomical structures. In: Proc MICCAI 2000, Pittsburgh, PA (2000)

15. Xu, C., Prince, J.: Snakes, shapes, and gradient vector flow. IEEE Transactions on Images Processing 7 (1998) 359-69

16. Guyon, I., Elisseeff, A.: An introduction to variable and feature selection. Journal of Machine Learning Research 3 (2003) 1157-82

17. Sandor, S., Leahy, R.: Surface-based labeling of cortical anatomy using a deformable database. IEEE Trans Med Imag 16 (1997) 41-54 